\title{
A NOTE ON REGULARIZATION BY LINEAR OPERATORS
}

\author{
NGUYEN BUONG
}

\begin{abstract}
The aim of this note is to give an improvement in our results of convergence rates of the regularized solutions for ill-posed operator equations involving monotone operators and in their convergence rates in combination with finite-dimensional approximations of reflexive Banach spaces .
\end{abstract}

Tóm tăt. Bài này trình bày một cải tiến tốt hơn cho tốc độ hội tụ cưa nghiệm hiệu chỉnh cưa bài toán không chính quy với toán tư đơn điệu và sự hội tụ đó cùng với việc xấp xỉ hũu hạn chiều không gian Banach.

\section{INTRODUCTION}

Let $X$ be a real reflexive Banach space and $X^{*}$ be dual space of $X$. For the sake of simplicity norms of $X$ and $X^{*}$ will be denoted by one symbol $\|$.$\| . We write \left\langle x^{*}, x\right\rangle$ instead of $x^{*}(x)$ for $x^{*} \in X^{*}$ and $x \in X$. Let $A$ be a monotone, continuous and bounded operator with domain of definition $D(A)=X$ and range $R(A) \subseteq X^{*}$.

We are interested in solving the ill-posed problem

$$
A(x)=f, \quad f \in R(A) .
$$

By ill-posedness we mean that the solutions of (1.1) do not depend continuously on the data $(A, f)$. To solve it we have to use stable methods. One of them was shown in [1]: Let $B$ is a linear operator such that

$$
\langle B x, x\rangle \geq m_{B}\|x\|^{2}, \forall x \in D(B), m_{B}>0, S_{0} \subset D(B), \overline{D(B)}=X,
$$

where $S_{0}$ denotes the set of solutions of (1.1), then the regularized equation

$$
A_{h}(x)+\alpha B x=f_{\delta},
$$

where $\left(A_{h}, f_{\delta}\right)$ are the approximations of $(A, f)$ with the following properties

$$
\begin{aligned}
\left\|A_{h}(x)-A(x)\right\| & \leq h g(\|x\|), \forall x \in X, \\
\left\|f_{\delta}-f\right\| & \leq \delta, h, \delta \rightarrow 0,
\end{aligned}
$$

$g(t)$ is a real and nondecreasing function with $g(0)=0, g(t) \rightarrow+\infty$, as $t \rightarrow+\infty$, and $A_{h}$ are also monotone, for every $\alpha>0$, has a unique solution $x_{h \delta}^{\alpha}$; if $h / \alpha, \delta / \alpha \rightarrow 0$, as $\alpha \rightarrow 0$, the sequence $\left\{x_{h \delta}^{\alpha}\right\}$ converges to $x_{1} \in S_{0}$,

$$
\left\langle B x_{1}, x-x_{1}\right\rangle \geq 0, \forall x \in S_{0} ;
$$

and the solution $x_{h \delta}^{\alpha}$ of $(1.2)$ can be approximated by solution of the finite-dimensional problem

$$
A_{h}^{n}(x)+\alpha B^{n} x=f_{\delta}^{n}
$$

with $A_{h}^{n}=P_{n}^{*} A_{h} P_{n}, B^{n}=P_{n}^{*} B P_{n}, f_{\delta}^{n}=P_{n}^{*} f_{\delta}$, under the conditions that

$$
X_{n} \subset D(B), X_{n} \subset X_{n+1}, B^{n} x=P_{n}^{*} B P_{n} x \rightarrow B x, \forall x \in D(B) .
$$

* This work was supported by the National Fundamental Research Program in Natural Sciences 
The convergence rates of the sequences $\left\{x_{h \delta}^{\alpha}\right\}$ and $\left\{x_{h \delta}^{\alpha n}\right\}$, where $x_{h \delta}^{\alpha n}$ denotes the solution of (1.3), are given by (see [1]).

Theorem 1.1. Assume that the following conditions hold:

(i) $A$ is Frćhet differentiable in some neighbourhood $U\left(S_{0}\right)$ of $S_{0}$.

(ii) There exists a constant $L>0$ such that

$$
\left\|A^{\prime}(x)-A^{\prime}(y)\right\| \leq L\|x-y\|, \forall x \in S_{0}, y \in U\left(S_{0}\right) .
$$

(iii) There exists an element $v \in D(B)$ such that

$$
A^{\prime}\left(x_{1}\right)^{*} v=B x_{1} .
$$

(iv) $L\|v\|<2 m_{B}$.

Then, if $\alpha$ is chosen as $\alpha \sim(h+\delta)^{\mu}, 0<\mu<1$, we obtain

$$
\left\|x_{h \delta}^{\alpha}-x_{1}\right\|=O\left((h+\delta)^{\theta}\right), \theta=\min \{1-\mu, \mu / 2\} .
$$

Remark: $\theta_{\max }=1 / 3$, when $\mu=2 / 3$.

Set

$$
\begin{aligned}
& \beta_{n}=\left\|P_{n}^{*} B P_{n} x_{1}-B x_{1}\right\|, \\
& \gamma_{n}=\left\|\left(I-P_{n}\right) x_{1}\right\| .
\end{aligned}
$$

Theorem 1.2. Let the following conditions hold:

(i) Conditions (i) - (iv) of Theorem 2.1 are fulfilled.

(ii) $\alpha$ is chosen as $\alpha \sim\left(h+\delta+\gamma_{n}\right)^{\mu}+\beta_{n}$.

Then

$$
\left\|x_{h \delta}^{\alpha n}-x_{1}\right\|=O\left(\left(h+\delta+\gamma_{n}\right)^{\theta}+\beta_{n}^{1 / 2}\right)
$$

where $\theta=\min \{1-\mu, \mu / 2\}$.

In this note, by using the approach in $[2]$ we can prove that the sequences $\left\{x_{h \delta}^{\alpha}\right\}$ and $\left\{x_{h \delta}^{\alpha n}\right\}$ converge with faster rates.

\section{RESULTS}

Theorem 2.1. Suppose that the following conditions hold:

(i) A is twice-Fréchet differentiable with $\left\|A^{\prime \prime}\right\| \leq M, M$ is a positive constant.

(ii) There exists an element $v \in D(B), B v \neq 0$, such that

$$
A^{\prime}\left(x_{1}\right) v=B x_{1} \text {. }
$$

(iii) $M\|v\|<2 m_{B}$.

Then, if $\alpha$ is chosen such that $\alpha \sim(h+\delta)^{\mu}, 0<\mu<1$, we have

$$
\left\|x_{h \delta}^{\alpha}-x_{1}\right\|=O\left((h+\delta)^{\theta}\right), \theta=\min \{1-\mu, \mu\} .
$$

Proof. From (1.1) and (1.2) it follows that

Set

$$
A\left(x_{h \delta}^{\alpha}\right)-A\left(x_{1}\right)+\alpha B\left(x_{h \delta}^{\alpha}-x_{1}\right)=f_{\delta}-f_{0}+A\left(x_{h \delta}^{\alpha}\right)-A_{h}\left(x_{h \delta}^{\alpha}\right)-\alpha B x_{1} .
$$

$$
P_{h \delta}^{\alpha}=\int_{0}^{1} A^{\prime}\left(x_{1}+t\left(x_{h \delta}^{\alpha}-x_{1}\right)\right) d t+\alpha B .
$$

It is easy to see that $P_{h \delta}^{\alpha}$ has the inversion $P_{h \delta}^{\alpha(-1)}$ with $\left\|P_{h \delta}^{\alpha(-1)}\right\| \leq 1 /\left(m_{B} \alpha\right)$. And, we have 


$$
\left\|x_{h \delta}^{\alpha}-x_{1}\right\| \leq\left(\delta+h g\left(\left\|x_{h \delta}^{\alpha}\right\|\right)\right) /\left(m_{B} \alpha\right)+\alpha\left\|_{h \delta}^{\alpha(-1)} B x_{1}\right\| .
$$

On the other hand,

$$
\begin{aligned}
\alpha\left\|P_{h \delta}^{\alpha(-1)} B x_{1}\right\| & =\alpha\left[\left\|P_{h \delta}^{\alpha(-1)}\left(P_{h \delta}^{\alpha}+A^{\prime}\left(x_{1}\right)-P_{h \delta}^{\alpha}\right) v\right\|\right] \\
& \leq \alpha\|v\|+\alpha \| P_{h \delta}^{\alpha(-1)}\left(P_{h \delta}^{\alpha}-A^{\prime}\left(x_{1}\right) v \|\right. \\
& \leq \alpha\left(\|v\|+\frac{\|B v\|}{m_{B}}\right)+\left\|\left(\int_{0}^{1} A^{\prime}\left(x_{1}+t\left(x_{h \delta}^{\alpha}-x_{1}\right)\right) d t-A^{\prime}\left(x_{1}\right)\right) v / m_{B}\right\| \\
& \leq \alpha\left(\|v\|+\frac{\|B v\|}{m_{B}}\right)+M\|v\|\left\|x_{h \delta}^{\alpha}-x_{1}\right\| /\left(2 m_{B}\right) .
\end{aligned}
$$

Therefore,

$$
\left(1-\frac{M\|v\|}{2 m_{B}}\right)\left\|x_{h \delta}^{\alpha}-x_{1}\right\| \leq\left(\delta+h g\left(\| x_{h \delta}^{\alpha} !\right)\right) /\left(m_{B} \alpha\right)+\alpha\left(\|v\|+\frac{\|B v\|}{m_{B}}\right) .
$$

Consequently,

$$
\left\|x_{h \delta}^{\alpha}-x_{1}\right\| \leq O\left((h+\delta)^{\theta}\right) .
$$

Hence,

$$
\left\|x_{h \delta}^{\alpha}-x_{1}\right\|=O\left((h+\delta)^{\theta}\right)(\text { see }[2]) .
$$

Remark: With $\mu=1 / 2$ the parameter $\theta$ achieves the maximal value $1 / 2$.

Set

$$
\tilde{\beta}_{n}=\max \left\{\beta_{n},\left\|B^{n} v-B v\right\|\right\} .
$$

Theorem 2.2. Suppose that conditions (i)-(iii) of Therem 2.1 hold and $\alpha$ is chosen such that $\alpha \sim\left(h+\delta+\gamma_{n}\right)^{\mu}+\tilde{\beta}_{n}, \quad 0<\mu<1$. Then we have

$$
\left\|x_{h \delta}^{\alpha n}-x_{1}\right\|=O\left(\left(h+\delta+\gamma_{n}\right)^{\theta}+\tilde{\beta}_{n}\right), \theta=\min \{1-\mu, \mu\} .
$$

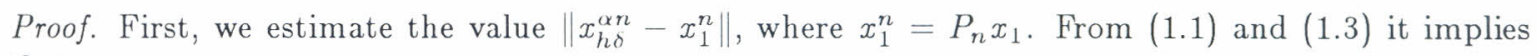
that

$$
\begin{aligned}
A^{n}\left(x_{h \delta}^{\alpha n}\right)-A^{n}\left(x_{1}^{n}\right)+\alpha B^{n}\left(x_{h \delta}^{\alpha n}-x_{1}^{n}\right)= & f_{\delta}^{n}-f^{n}-\alpha B^{n} x_{1}^{n}+P_{n}^{*}\left(A\left(x_{1}\right)-A\left(x_{1}^{n}\right)\right) \\
& +A^{n}\left(x_{h \delta}^{\alpha n}\right)-A_{h}^{n}\left(x_{h \delta}^{\alpha n}\right)
\end{aligned}
$$

where $A^{n}=P_{n}^{*} A P_{n}$, and $f^{n}=P_{n}^{*} f$.

Set

$$
P_{h \delta}^{\alpha n}=\int_{0}^{1} P_{n}^{*} A^{\prime}\left(x_{1}^{n}+t\left(x_{h \delta}^{\alpha n}-x_{1}^{n}\right)\right) d t+\alpha B^{n} .
$$

Clearly, the operator $P_{h \delta}^{\alpha n}$ is linear, bounded and monotone from $X_{n}$ onto $X_{n}^{*}$ with $\left\|P_{h \delta}^{\alpha n(-1)}\right\| \leq$ $1 /\left(m_{B} \alpha\right)$. Since

$$
\begin{gathered}
\left\|P_{h \delta}^{\alpha n(-1)} P_{n}^{*}\left(f_{\delta}-f\right)\right\| \leq \delta /\left(m_{B} \alpha\right), \\
\left\|P_{h \delta}^{\alpha n(-1)} P_{n}^{*}\left(A_{h}\left(x_{h \delta}^{\alpha n}\right)-A\left(x_{h \delta}^{\alpha n}\right)\right)\right\| \leq \frac{h}{m_{B} \alpha} g\left(\left\|x_{h \delta}^{\alpha n}\right\|\right), \\
\left\|P_{h \delta)}^{\alpha n(-1)} P_{n}^{*}\left(A\left(x_{1}\right)-A\left(x_{1}^{n}\right)\right)\right\| \leq \\
\leq\left\|P_{h \delta}^{\alpha n(-1)}\left[A^{\prime}\left(x_{1}\right)\left(I-P_{n}\right) x_{1}+A^{\prime \prime}\left(x_{1}+\tau\left(P_{n}-I\right) x_{1}\right)\left(I-P_{n}\right) x_{1}\left(I-P_{n}\right) x_{1}\right]\right\| \\
\leq \gamma_{n}\left\|A^{\prime}\left(x_{1}\right)\right\| /\left(m_{B} \alpha\right)+\gamma_{n}^{2} M /\left(2 m_{B} \alpha\right) \\
\leq O\left(\gamma_{n} / \alpha\right),
\end{gathered}
$$




$$
\begin{gathered}
\alpha\left\|P_{h \delta}^{\alpha n(-1)} B^{n} x_{1}^{n}\right\| \leq \alpha\left\|P_{h \delta}^{\alpha n(-1)} P_{n}^{*}\left(B^{n} x_{1}-B x_{1}\right)+P_{h \delta}^{\alpha n(-1)} P_{n}^{*} B x_{1}\right\| \\
\leq \beta_{n} / m_{B}+\alpha\left\|P_{h \delta}^{\alpha n(-1)} B x_{1}\right\|, \\
\alpha\left\|P_{h \delta}^{\alpha n(-1)} P_{n}^{*} B x_{1}\right\|=\alpha\left\|P_{h \delta}^{\alpha n(-1)} P_{n}^{*}\left(P_{h \delta}^{\alpha n}+A^{\prime}\left(x_{1}\right)-P_{h \delta}^{\alpha n}\right) v\right\|, \\
\alpha\|v\|+\frac{M\|v\| \gamma_{n}}{m_{B}}+\alpha\left\|P_{h \delta}^{\alpha n(-1)} P_{n}^{*}\left(P_{h \delta}^{\alpha n}-A^{\prime}\left(x_{1}^{n}\right)\right) v\right\| \leq \\
\leq \alpha\|v\|+\frac{M\|v\| \gamma_{n}}{m_{B}}+\frac{\alpha \tilde{\beta}_{n}+\alpha\|B v\|}{m_{B}}+\alpha\left\|P_{h \delta}^{\alpha n(-1)}\left(\int_{0}^{1} A^{\prime}\left(x_{1}^{n}+t\left(x_{h \delta}^{\alpha n}-x_{1}^{n}\right)\right) d t-A^{\prime}\left(x_{1}^{n}\right)\right) v\right\| \\
\leq \alpha\|v\|+\frac{M\|v\| \gamma_{n}}{m_{B}}+\frac{\alpha\left(\tilde{\beta}_{n}+\|B v\|\right)}{m_{B}}+\frac{M\|v\|\left\|_{h \delta}^{\alpha n}-x_{1}^{n}\right\| .}{2 m_{B}} \| x_{h \delta}
\end{gathered}
$$

Therefore,

$$
\left\|x_{h \delta}^{\alpha n}-x_{1}^{n}\right\| \leq O\left(\left(h+\delta+\gamma_{n}\right) / \alpha+\alpha+\tilde{\beta}_{n}+\gamma_{n}\right) .
$$

Hence (see $[2]$ ),

$$
\left\|x_{h \delta}^{\alpha n}-x_{1}\right\|=O\left(\left(h+\delta+\gamma_{n}\right)^{\theta}+\tilde{\beta}_{n}\right) .
$$

\section{REFEREN CES}

[1] Nguyen Buong, Regularization by linear operators, Acta Math. Vietnam. 21 (1) (1996) 135-145.

[2] Bakushinskii A. and Goncharsky A., Ill-Posed Problems: Theory and Applications, Dordrecht Boston - London: Kluwer Acad. Publishers, 1994.

Received February 16, 2000 Revised March 4, 2001

Institute of Information Technology 